\title{
Diagnosis and management of small pulmonary atypical carcinoid tumor associated with Cushing syndrome
}

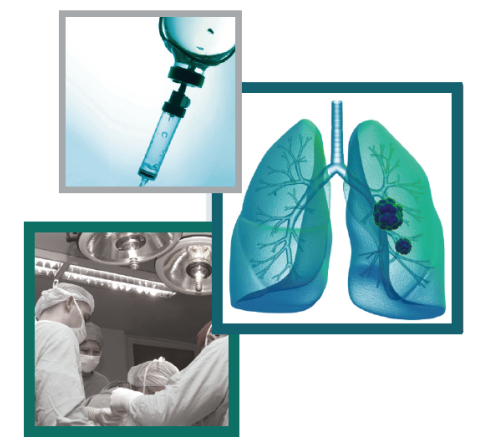

\author{
Dathe Z Benissan-Messan ${ }^{1}$, Robert E Merritt ${ }^{1}$, Konstantin Shilo ${ }^{1}$, Desmond M D'Souza ${ }^{1}$ \& \\ Peter J Kneuertz*,1 (iD \\ ${ }^{1}$ Thoracic Surgery Division, Department of Surgery, The Ohio State University Wexner Medical Center, Columbus, OH 43210, USA \\ *Author for correspondence: Peter.Kneuertz@osumc.edu
}

\section{Practice points}

- Ectopic adrenocorticotropic hormone syndrome is rare and identification of its source can be difficult.

- The most common location of ectopic adrenocorticotropic hormone producing tumor is the lung.

- The optimal treatment of ectopic adrenocorticotropic hormone syndrome (EAS) is surgical resection.

- Steroid inhibitors are the mainstay of treatment to gain control of hypercortisolemia until a culprit is identified.

- Prognosis is determined by tumor histology and severity of hypercorticolism.

- Early consideration should be given to obtaining Ga-DOTATATE PET imaging in cases of occult disease to decrease the time to tumor localization and mitigate morbidity.

Ectopic adrenocorticotropic hormone (ACTH) syndrome is rare and identification of its source is often challenging. We report the case of an ectopic Cushing syndrome in a young adult male secondary to an occult ACTH producing atypical carcinoid tumor. Extensive biochemical and imaging workup was unrevealing. The diagnosis was aided by Ga-DOTA PET scan demonstrating a suspicious left upper lobe lung nodule. The patient underwent video-assisted thoracoscopic exploration with wedge resection and mediastinal lymphadenectomy of a T2aN2M0 atypical carcinoid, resulting in the normalization of ACTH levels and complete resolution of symptoms. The role of a Ga-DOTA PET scan in diagnosing pulmonary carcinoid tumors and their management are discussed.

First draft submitted: 23 March 2020; Accepted for publication: 5 August 2020; Published online: 14 September 2020

Keywords: atypical carcinoid $\bullet$ ectopic ACTH syndrome $\bullet$ ectopic Cushing syndrome $\bullet$ Ga-DOTA PET $\bullet$ hypercortisolemia $\bullet$ neuroendocrine tumors $\bullet$ pulmonary carcinoid $\bullet$ video-assisted thoracoscopic surgery

The lung is the second most common site for neuroendocrine tumors (NETs), accounting for $25 \%$ of all NETs and $1-2 \%$ of all lung cancers [1]. Atypical Carcinoids (AC) are a rare subtype of NETs which represent $0.2 \%$ of thoracic malignancies with a $20 \%$ chance of distant spread [1]. About $40 \%$ of patients presenting with ectopic Cushing syndrome are found to have pulmonary carcinoids [2]. These hormonally active tumors are often small but can be associated with a more aggressive behavior relative to typical carcinoid tumors and often present a diagnostic challenge [3,4]. Here, we report the case of an ectopic Cushing syndrome in a young adult patient caused by an adrenocorticotropic hormone (ACTH) producing AC tumor, which highlights the diagnostic role of ${ }^{68} \mathrm{Ga}$ DOTATATE (GA-DOTA) PET scan and surgical management.

\section{Case report}

A 27-year-old white male, who was previously in excellent health and performance status, was admitted to our hospital as a transfer from a referring hospital with new onset of acute uncontrolled diabetes requiring insulin, persistent hypokalemia, weakness and a suspected diagnosis of Cushing's syndrome. MRI of the adrenals and brain showed normal appearing adrenal and pituitary glands. Low- and high-dose dexamethasone tests resulted in elevated morning cortisol and adrenocorticotropic hormone (ACTH) level (Table 1). His laboratory evaluation revealed hypothyroidism, but a thyroid ultrasound was also normal. Computed tomography (CT) images of his 
Table 1. Summary of hormone analysis work up.

\begin{tabular}{|l|l|}
\hline Plasma ACTH & $325 \mathrm{pg} / \mathrm{ml}(9-50)$ \\
\hline $24 \mathrm{~h}$ Urine cortisol & $21,108 \mathrm{mcg}(3.5-45)$ \\
\hline Random cortisol & $139.02 \mathrm{mcg} / \mathrm{dl}(0.09-22.40)$ \\
\hline AM cortisol with $1 \mathrm{mg}$ dexamethasone & $108.03 \mathrm{mcg} / \mathrm{dl}(0.09-22.40)$ \\
\hline AM cortisol with $8 \mathrm{mg}$ dexamethasone & $110.66 \mathrm{mcg} / \mathrm{dl}(0.09-22.40)$ \\
\hline TSH & $0.204 \mathrm{ulU} / \mathrm{ml}(0.55-4.78)$ \\
\hline T4 Free & $0.84 \mathrm{ng} / \mathrm{dl}(0.89-1.76)$ \\
\hline Total T3 & $0.54 \mathrm{ng} / \mathrm{dl}(0.60-1.81)$ \\
\hline C-peptide & $2.4 \mathrm{ng} / \mathrm{ml}(0.2-2.7)$ \\
\hline Renin, direct & $4.3 \mathrm{pg} / \mathrm{ml}(4.2-52.2)$ \\
\hline Aldosterone & $3.8 \mathrm{ng} / \mathrm{dl}(<35.4)$ \\
\hline IFG-1 & $52 \mathrm{ng} / \mathrm{ml}(83.6-259)$ \\
\hline Testosterone & $45 \mathrm{ng} / \mathrm{dl}(87-814)$ \\
\hline ACTH: Adrenocorticotropic hormone; TSH: Thyroid-stimulating hormone. \\
\hline
\end{tabular}

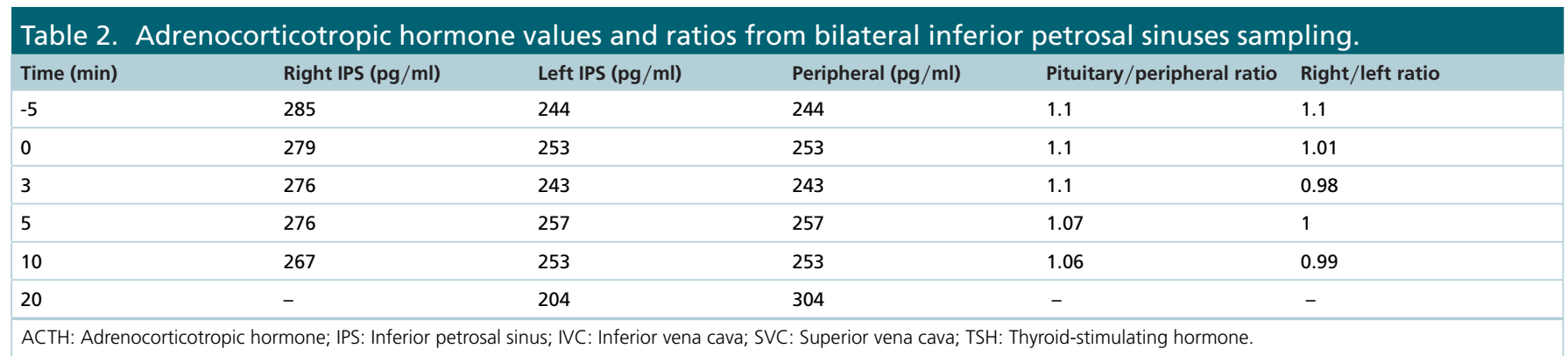

chest revealed bilateral pleural effusions and associated atelectasis, without evidence of a nodule or mass. Although the constellation of his symptoms at presentation was suspicious for a diagnosis of ectopic ACTH-induced Cushing syndrome, identification of the culprit proved difficult. Since he had secondary hypothyroidism as well as low IGF-1 and low testosterone level, he was initially suspected to have a pituitary lesion. A repeat high-resolution pituitary MRI was unremarkable. Following an episode of gastrointestinal bleed during his hospitalization, the diagnostic focus shifted to the identification of an intra-abdominal ACTH producing mass. A liver protocol scan did not reveal any masses and showed a short segment focus of jejunojejunal intussusception. A follow-up MRI enterography and colonoscopy were negative for any gastrointestinal neoplasm. An endoscopic ultrasound of his pancreas was also obtained and was unremarkable. An octreotide scan revealed no definite evidence of somatostatin receptor avid lesion. Neurosurgery was then consulted and the patient underwent bilateral inferior petrosal sinus sampling. Central-to-peripheral ACTH gradients were less than two bilaterally (Table 2). After a month-long hospitalization and several inconclusive tests, a Ga-DOTATATE PET was obtained, which revealed a small lung soft tissue density in the tip of the lingula of the left upper lobe in the cardiophrenic sulcus with mild radiotracer activity (Figure 1).

At the time of his evaluation in thoracic surgery clinic, he had endorsed over $40 \mathrm{lbs}$ of weight loss despite significant peripheral edema due to newly developed congestive heart failure and was severely deconditioned, unable to ambulate and fully reliant on a wheelchair for mobility. Hypercortisolemia was being managed with Ketoconazole. Following a thorough evaluation, a diagnostic video-assisted thoracoscopic surgery was offered for wedge resection of a suspected hormonally active pulmonary NET. The patient was not deemed a candidate for lobectomy given his poor performance status and overall condition. During surgery, a nodule was palpated in the most anterior tip of the lingula and a generous wedge resection was performed. Intraoperative frozen pathology assessment revealed carcinoid tumor. A complete mediastinal lymph node dissection was performed. Postoperatively, his ACTH/cortisol levels normalized on postoperative day 1 (Figure 2). He was maintained on corticosteroid and mineralocorticoid replacement postoperatively, which were titrated based on AM cortisol measurements. Pathology revealed a $3.6 \mathrm{~cm}$ moderately differentiated $\mathrm{AC}$ tumor involving the lingula with 1 mitosis per $\mathrm{mm}$ and a KI67 of 


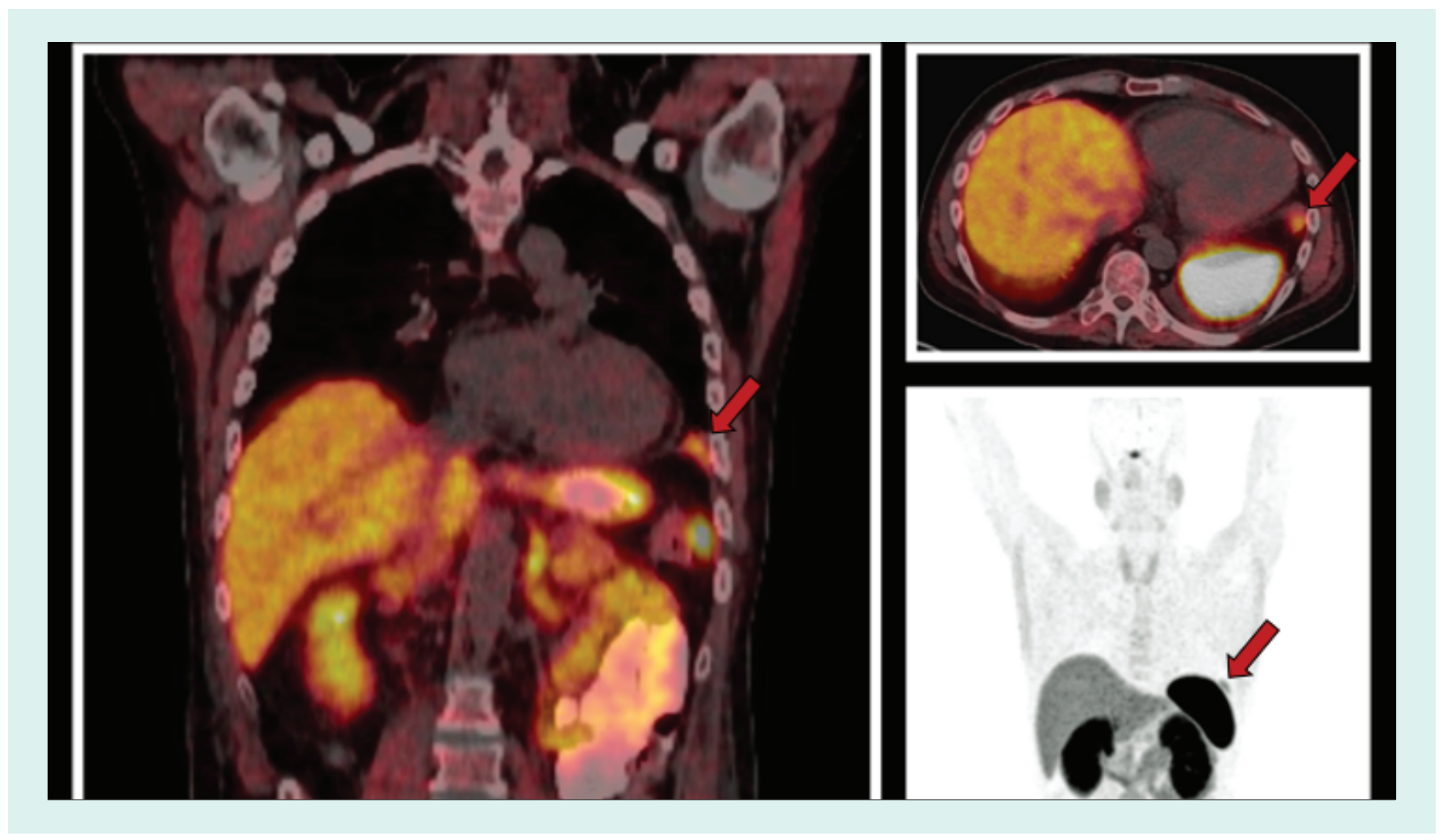

Figure 1. Ga-DOTA-somatostatin analogue PET demonstrating a avid nodule in the lingula of the left upper lobe in the cardiophrenic sulcus.

$1 \%$, but lympho-vascular and visceral-pleural invasion (Figure 3). Two mediastinal lymph nodes were positive in level 5 (AJCC stage pT2a N2). His case was reviewed in a multidisciplinary tumor board setting. It was decided against further lobectomy or adjuvant chemotherapy given the risk in the setting of the patients' overall poor condition. Following discharge from the hospital on day 4 , he continued with an inpatient physical rehabilitation program. By week two, he was able to ambulate independently with the help of a cane and was weaned off his oral diabetic medication. He was maintained on bisphosphonate treatment for glucocorticoid induced osteoporosis. The steroid replacement was tapered slowly over a three-month period. His symptom resolved completely, and his heart function returned to normal. He has remained in close follow-up and without evidence of recurrence for over two years after resection .

\section{Discussion}

The diagnosis of ectopic ACTH syndrome focuses on the differentiation of pituitary versus ectopic ACTH production after confirmation of endogenous hypercortisolism and demonstration of elevated plasma ACTH which excludes adrenal disease [5]. The lung is the most common source of ectopic ACTH [6]. Cushing syndrome manifests in 1-6\% of patients with pulmonary carcinoids [2]. The diagnosis of an occult ectopic ACTH producing nodule far to commonly presents a challenge to the clinician. The tumors implicated are often small and difficult to detect, which may delay the diagnosis and result in progression of disease [6]. CT, MRI and scintigraphic studies localize ACTH-secreting tumors in 70-90\% of cases [6]. Nineteen percent of ACTH-secreting tumors remain occult despite intensive investigation but are readily identified with Ga-DOTA somatostatin analogue (SSA) PET/CT [6]. Ga-DOTA-SSA PET/CT provides high sensitivity (88-93\%) and specificity (88-95\%) for the diagnosis and staging of carcinoid tumors [6]. Although contrasted CT scans are the gold standard for the diagnosis of carcinoids tumors, Ga-DOTA-SSA PET may be a great modality to localize occult disease $[1,2,6]$. The workflow and clinical management guidelines in the evaluation of EAS are detailed by Alexandraki et al. and Hayes et al. [5,6]. Early consideration in the management of occult EAS ought to be considered as they have better resolution, decreased scanning time and higher binding affinity to somatostatin receptors [7-9]. 


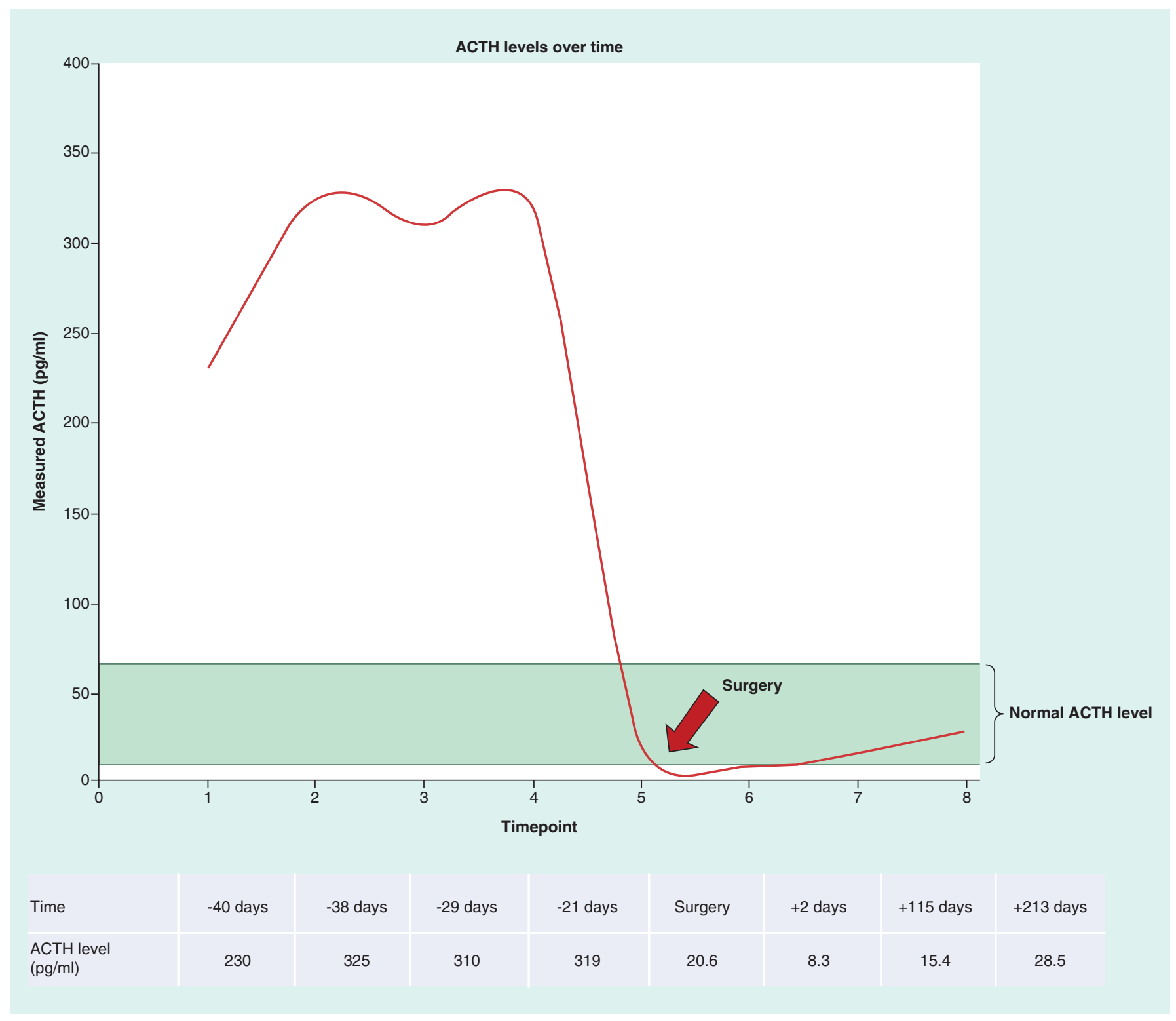

Figure 2. Perioperative adrenocorticotropic hormone levels demonstrating rapid normalization after surgery. Time on $x$-axis relative to surgery. Area shaded in green marks normal adrenocorticotropic hormone range in male patient.

ACTH: Adrenocorticotropic hormone.

ACs are a rare subtype of NETs with no established association with smoking and a predilection for middle aged patients [10]. Five-year overall survival varies from 50 to $80 \%$ and distant metastases are found in up to $50 \%$ of AC [10,11]. The most common sites of pulmonary carcinoid metastasis are mediastinal lymph nodes, followed by the liver and bones [2]. Surgery is the mainstay of treatment for ACs and should be considered for curative intent or symptoms control in patients with advanced disease. Resection of oligometastases is considered depending on the site of metastasis [1]. Systematic nodal dissection is advised for ACs since the tumors carry a 30-70\% risk of regional lymph nodes involvement [12]. Formal anatomical resection is recommended for atypical peripheral pulmonary carcinoids if feasible, as cases of regional recurrences have been noted up to and beyond 30 years from the original resection of the primary tumor [2]. Hormone levels are expected to normalize rapidly after resection (Figure 2) of secreting tumors and may be used to follow patients as an adjunct to imaging surveillance. Patients diagnosed with a low grade lung NET should undergo frequent follow-up after surgical resection. Although more 


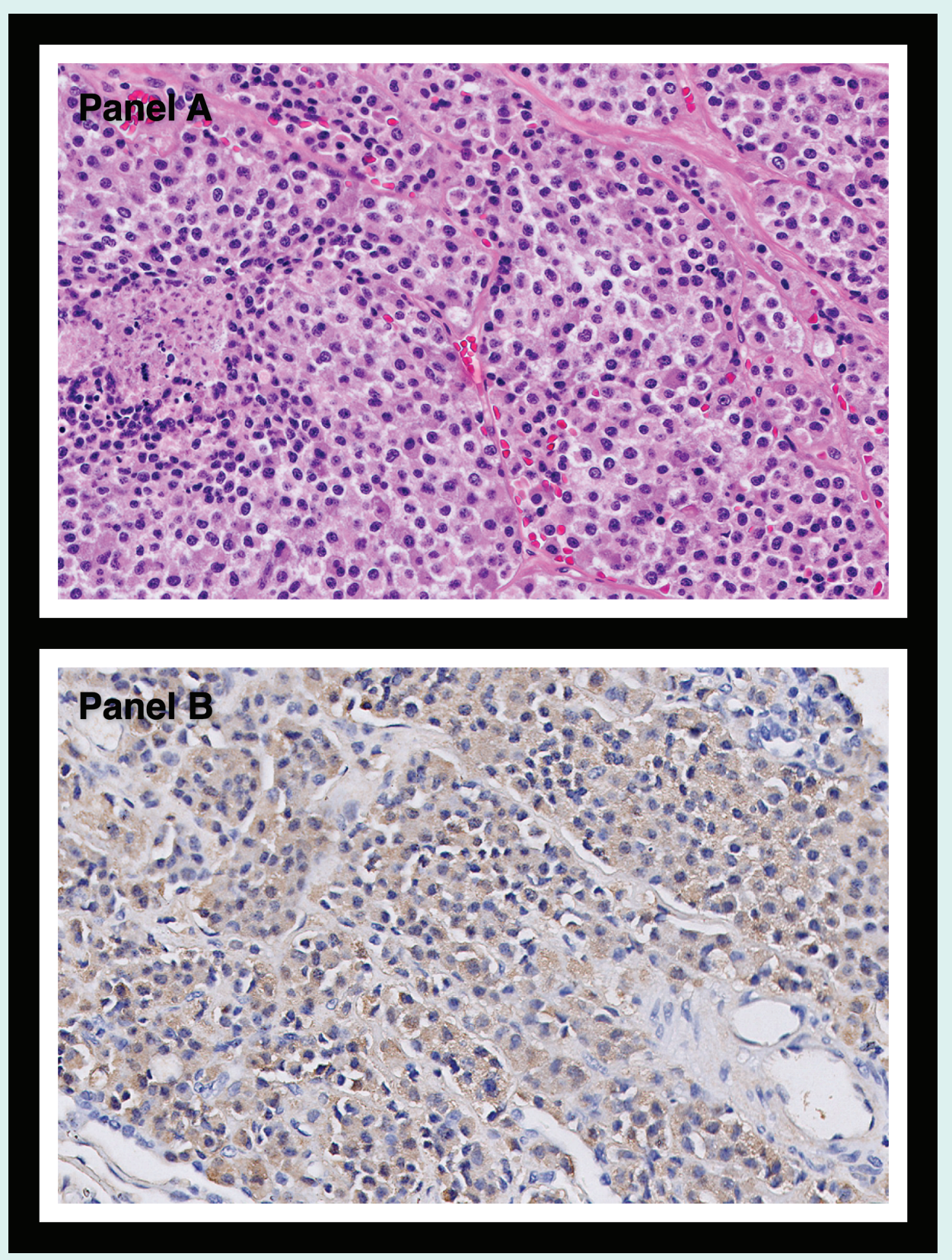

Figure 3. Pathological features of atypical carcinoid tumor. (A) Shows light microscopic morphology of the tumor with characteristic nested architecture and a focus of necrosis; Hematoxylin and eosin stain; original magnification $200 \times$. (B) Shows cytoplasmic adrenocorticotropic hormone expression; immunohistochemistry; original magnification $200 \times$.

guidelines are needed to establish a follow-up timeline, closer monitoring is generally recommended for ACs due to their aggressive nature.

For patients with unresectable or metastatic disease, SSAs are effective in management of symptoms and in slowing disease progression. SSAs can induce stabilization in 30-70\% of patients with well differentiated NETs [2]. mTOR inhibitors can be considered in advanced nonfunctional typical or AC [1]. There is no consensus on adjuvant therapy in pulmonary carcinoids after complete resection. Chemotherapy is often considered in ACs with progressive metastases when no other treatment options exit [1,2]. A recent study by Gosain et al. however, found 
that patients with node positive AC had no significant overall survival difference with adjuvant chemotherapy and that patients with stage I ACs had worse survival with adjuvant chemotherapy [11].

\section{Conclusion}

A high level of clinical suspicion should to be maintained in the evaluation of patients with ectopic Cushing syndrome. The most common location of ectopic ACTH producing tumor remains the lung. Early consideration should be given to obtaining Ga-DOTATATE PET imaging in cases of occult disease to decrease the time to tumor localization and cure with resection.

\section{Financial \& competing interests disclosure}

The authors have no relevant affiliations or financial involvement with any organization or entity with a financial interest in or financial conflict with the subject matter or materials discussed in the manuscript. This includes employment, consultancies, honoraria, stock ownership or options, expert testimony, grants or patents received or pending, or royalties.

No writing assistance was utilized in the production of this manuscript.

\section{Ethical conduct of research}

The authors state that they have obtained verbal and written informed consent from the patient/patients for the inclusion of their medical and treatment history within this case report.

Open access

This work is licensed under the Attribution-NonCommercial-NoDerivatives 4.0 Unported License. To view a copy of this license, visit http://creativecommons.org/licenses/by-nc-nd/4.0/

\section{References}

Papers of special note have been highlighted as: $\bullet$ of interest; $\bullet \bullet$ of considerable interest

1. Melosky B. Advanced typical and atypical carcinoid tumours of the lung: management recommendations. Curr. Oncol. 25(Suppl. 1), S86-S93 (2018).

- Highlights the factors that have to be taken into account in the management of neuroendocrine tumors.

2. Caplin ME, Baudin E, Ferolla P et al. Pulmonary neuroendocrine (carcinoid) tumors: European Neuroendocrine Tumor Society expert consensus and recommendations for best practice for typical and atypical pulmonary carcinoids. Ann. Oncol. 26(8), 1604-1620 (2015).

3. Boddaert G, Grand B, Le Pimpec-Barthes F, Cazes A, Bertagna X, Riquet M. Bronchial carcinoid tumors causing Cushing's syndrome: more aggressive behavior and the need for early diagnosis. Ann. Thorac. Surg. 94(6), 1823-1829 (2012).

4. Shrager JB, Wright CD, Wain JC, Torchiana DF, Grillo HC, Mathisen DJ. Bronchopulmonary carcinoid tumors associated with Cushing's syndrome: a more aggressive variant of typical carcinoid. J. Thorac. Cardiovasc. Surg. 114(3), 367-375 (1997).

5. Alexandraki KI, Grossman AB. The ectopic ACTH syndrome. Rev. Endocr. Metab. Disord. 11(2), 117-126 (2010).

6. Hayes AR, Grossman AB. The ectopic adrenocorticotropic hormone syndrome: rarely easy, always challenging. Endocrinol. Metab. Clin. North Am. 47(2), 409-425 (2018).

-. Highlights the difficulty in diagnosis EAS as well as the work up and management of the syndrome.

7. Gosain R, Mukherjee S, Yendamuri SS, Iyer R. Management of typical and atypical pulmonary carcinoids based on different established guidelines. Cancers 10(12), 510 (2018).

8. Haug AR, Cindea-Drimus R, Auernhammer CJ et al. The role of ${ }^{68} \mathrm{Ga}$-DOTATATE PET/CT in suspected neuroendocrine tumors. J. Nucl. Med. 53(11), 1686-1692 (2012).

- $\quad{ }^{68}$ Ga-DOTATATE PET/CT is highly accurate in patient with suspected neuroendocrine tumors.

9. Srirajaskanthan R, Kayani I, Quigley AM, Soh J, Caplin ME, Bomanji J. The role of ${ }^{68}$ Ga-DOTATATE PET in patients with neuroendocrine tumors and negative or equivocal findings on 111In-DTPA-octreotide scintigraphy. J. Nucl. Med. 51(6), 875-882 (2010).

10. Hung YP. Neuroendocrine tumors of the lung: updates and diagnostic pitfalls. Surg. Pathol. Clin. 12(4), 1055-1071 (2019).

11. Gosain R, Groman A, Yendamuri SS, Iyer R, Mukherjee S. Role of adjuvant chemotherapy in pulmonary carcinoids: an NCDB analysis. Anticancer Res. 39(12), 6835-6842 (2019).

12. Okoye CC, Jablons DM, Jahan TM, Kukreja J, Cardozo S, Yom SS. Divergent management strategies for typical versus atypical carcinoid tumors of the thoracic cavity. Am. J. Clin. Oncol. 37(4), 350-355 (2014). 